\title{
S100A4 Expression is Closely Linked to Genesis and Progression of Glioma by Regulating Proliferation, Apoptosis, Migration and Invasion
}

\author{
Ting Jin ${ }^{1}$, Zhuo Zhang ${ }^{2}$, Xue-Feng Yang ${ }^{3}$, Jun-Sheng Luo ${ }^{3 *}$
}

\begin{abstract}
Background: The calcium-binding S100A4 protein is involved in epithelial to mesenchymal transition, oncogenic transformation, angiogenesis, cytoskeletal integrity, mobility and metastasis of cancer cells. This study aimed to clarify the roles of S100A4 in genesis and progression of glioma. Materials and Methods: S100A4 expression was examined by real-time RT-CPR and Western blot in glioma and paired normal brain tissue ( $n=69)$, and compared with clinicopathological parameters of tumors. In addition, glioma U251 cells transfected with an S100A4-expressing plasmid were examined for proliferation by MTT, apoptosis by Annexin V-FITC, and migration and invasion with Transwell chambers. Results: Increased S100A4 mRNA expression was found in gliomas, compared with paired non-tumor tissue $(p<0.001)$. Gradual elevation of overexpression of S100A4 was observed with increasing glioma grade $(p<0.001)$. Astrocytoma showed lower S100A4 mRNA expression than oligodendrogliomas, with glioblastomas having highest values $(p<0.001)$. Similar results were obtained for S100A4 protein, a positive link being found between mRNA and protein expression in gliomas $(p<0.001)$. There was higher growth, lower apoptosis, stronger migration and invasion of $\mathrm{S100A4}$ transfectants than control and mock transfected cells $(p<0.001)$. Conclusions: These findings indicate that up-regulated S100A4 expression is positively linked to pathogenesis, progression and histogenesis of glioma by modulating proliferation, apoptosis, migration and invasion.
\end{abstract}

Keywords: Glioma - S100A4 - pathogenesis - progression - aggressiveness

Asian Pac J Cancer Prev, 16 (7), 2883-2887

\section{Introduction}

Glioma arises from the glial cells of the brain, which make up about $30 \%$ of all brain and central nervous system tumors and $80 \%$ of all malignant brain tumors. Histologically, it includes astrocytomas, oligodendrogliomas, glioblastoma and etc (Trabelsi, 2014). There is a tendency toward a higher incidence of gliomas in highly developed and industrialized countries because occupations, environmental carcinogens, and diets have been reported to be associated with an elevated glioma risk. With the exception of pilocytic astrocytomas (WHO grade I), the prognosis of glioma patients is still poor, especially glioblastoma (Schwartzbaum et al., 2006). Therefore, it is essential for the glioma to find out good and effective molecular markers and gene therapy targets.

Acidic S100 genes include about 25 members and cluster on human chromosome 1q21, whose encoding proteins of low molecular weight $(10-12 \mathrm{kDa})$ contain 2 EF-hand calcium-binding helix- loop-helix motifs and might be dissolved in $100 \%$ saturated ammonium sulfate. S100A4 (Mts1, metastasis-associated gene; p9Ka; 18A2,
pEL98, 42A, CAPL, and calvasculin) protein belongs to the S100 protein family (Jenkinson et al., 2004; Boye and Maelandsmo, 2010; Mishra et al., 2012). PI3K/Akt/ mTOR/ p70S6K, MAPK/ERK and hypoxia pathways mediate S100A4- induced cell motility in cancer cells by interaction with as F-actin, myosin-IIA, tropomyosin, and the heavy chain of nonmuscle myosin II (Jenkinson et al., 2004; Dahlmann et al., 2014; Wang et al., 2014). Xu et al.(2014) found that sonic Hedgehog-Gli1 signaling pathway regulates the epithelial mesenchymal transition (EMT) by regulating S100A4 in pancreatic cancer cells. Additionally, S100A4 might reduce p53 phosphorylation and subsequently suppress the transcription of p53 target genes, including p21/WAF, Bax, thrombospondin-1, and Mdm-2 (Zhao et al., 2013). Down-regulated S100A4 expression by antisense or anti-ribozyme suppressed the metastatic potential of tumor cells in xenograft models of lung carcinoma and osteosarcoma (Maelandsmo et al., 1996; Takenaga et al., 1997). S100A4 knockdown reduced tumorigenesis and metastasis of osteosarcoma cells by suppressing matrix metalloproteinase-9 (Zhang et al., 2014). There was no difference in breast carcinogenesis 
Ting Jin et al

between transgenic mice of S100A4 overexpression and wild-type mice, but high metastasis was observed in transgenic mice (Simpson et al., 2003).

To clarify the expression status and clinicopathological significances of S100A4 expression, we aimed to observe S100A4 expression in various subtypes of gliomas at both protein and mRNA levels and compared its expression with clinicopathological features. Finally, we ectopically ovexpressed S100A4 expression in glioma cell and observed such cellular phenotypes as proliferation, apoptosis, migration and invasion to clarify the molecular mechanisms of its biological effects.

\section{Materials and Methods}

\section{Subjects and pathology}

Glioma and matched normal brain tissues were collected from surgical specimens in Department of Neurosurgery, The First Affiliated Hospital of Liaoning Medical University from January 2008 to January 2013. These tumors included 49 astrocytomas, 11 oligodendrogliomas and 9 glioblastomas. The part of tissues was subjected to routine pathological examination to confirm their histological diagnosis and other microscopic characteristics. The part of some samples were frozen in liquid nitrogen and stored at $-80 \mathrm{oC}$ until protein and RNA extraction by homogenization. Both subtyping and grading for each glioma were evaluated according to WHO classification (Louis et al., 2007). None of the patients underwent chemotherapy, radiotherapy or adjuvant treatment before surgery. They all provided consent for use of tumor tissue for clinical research and the University Ethical Committee of Liaoning Medical University approved the research protocol. We followed up the patients by consulting their case documents and through telephone.

\section{Cell culture and transfection}

Glioma cell line, U251 come from Cell bank of Chinese Academy of Sciences, Shanghai, China, respectively and was maintained in RPMI 1640 medium supplemented with $10 \%$ fetal bovine serum (FBS), 100 units $/ \mathrm{mL}$ penicillin, and $100 \mu \mathrm{g} / \mathrm{mL}$ streptomycin in a humidified atmosphere of $5 \% \mathrm{CO}_{2}$ at $37^{\circ} \mathrm{C}$.

$\mathrm{S} 100 \mathrm{~A} 4$ gene was amplified by forward primer: 5' - GGGG TA C C C C AT G GCG T G C C C T C T G GAGAAGG-3' and backward primer 5' CGGGATCCCGTCATTTCTTCCTGGGCTGCTTA-3' using the template cDNA from U251 cells. The PCR products were inserted into pcDNA3.1 (Clontech, USA) between KpnI and BamHI. U251 cells were transfected with pcDNA3.1-S100A4 or pcDNA3.1 vector as mock at $24 \mathrm{~h}$ after seeding on dishes, and selected by G418 according to the manufacturer's instructions (QIAGEN, USA) with final collection of three monoclones.

\section{Real-time RT-PCR}

Total RNA was extracted by TRIzol, and cDNAs were reverse transcribed by RevertAid TM reverse transcriptase (Takara). Real-time PCR was carried out using the ABI PRISM 7500 Sequence detection system (Applied Biosystems). The primers for GAPDH (135bp, 201-335, NM_002046.3) were 5'-CAATGACCCCTTCATTGACC-3' (sense) and 5'TGGAAGATGGTGATGGGATT-3' (antisense). The primers for S100A4 (212bp, 333-525, NM_005978.3) were 5'-TCCAAGAGTAC TGTGTCTTC-3' (sense) and 5'-TATTGAACTTGCTCAGCATC-3' (antisense). Expression of GAPDH was used to normalize that of the target genes. Each assay was done in triplicate, the average was calculated, and the expression level of S100A4 was expressed as $2^{-\Delta \mathrm{Ct}}$, where $\Delta \mathrm{Ct}=\mathrm{Ct}(\mathrm{S} 100 \mathrm{~A} 4)-\mathrm{Ct}(\mathrm{GAPDH})$.

\section{Western blot}

Protein was extracted by homogenization in RIPA lysis buffer and assayed by BAC method. Denatured protein was separated on an SDS-polyacrylamide gel (15\% acrylamide) and transferred to Hybond membrane (Amersham, Amersham, Germany), which was then blocked overnight in 5\% skim milk in TBST $(10 \mathrm{mmol} / \mathrm{L}$ Tris- $\mathrm{HCl}, 150 \mathrm{mmol} / \mathrm{L} \mathrm{NaCl}, 0.1 \%$ Tween 20). For immunobloting, the membrane was incubated for $1 \mathrm{~h}$ with the rabbit antibody against S100A4 (Novus Biologicals, USA, 1: 500). Then, it was rinsed by TBST and incubated with anti-rabbit or anti-mouse IgG conjugated to horseradish peroxidase (DAKO, Carpinteria, CA93013, USA, 1:1000) for $1 \mathrm{~h}$. Bands were visualized with LAS 4010 (GE, Japan) by ECL-Plus detection reagents (Santa Cruz, USA). After that, membrane was washed with WB Stripping Solution (pH2-3, Nacalai, Tokyo, Japan) for 1 hour and treated as described above except anti-GAPDH antibody (Sigma, 1:10000) as an internal control antibody. Densitometric quantification of S100A2 protein in gastric samples was performed with GADPH control using Scion Image software (Scion Corporation, Frederick, MD, USA).

\section{Cell viability}

In brief, $2.5 \times 10^{3}$ cells/well were seeded on 96-well plate and allowed to adhere. At different time points, $10 \mu \mathrm{L}$ MTT solution $(10 \mathrm{mg} / \mathrm{ml})$ was added into each well of the plate and the plates were incubated for $3 \mathrm{~h}$ in the incubator. The culture fluid was removed and MTT formazan was dissolved in $100 \mu \mathrm{L}$ DMSO. Finally, the 96-well plate was read at $590 \mathrm{~nm}$.

\section{Apoptosis assay}

Flow cytometry was performed with PI and FITC-labeled annexin V (Kagen, China) to detect phosphatidylserine externalization as an endpoint indicator of apoptosis according to the manufacturer's instructions.

\section{Cell migration and invasion assays}

For migration assay, $2.5 \times 10^{5}$ cells were resuspended in serum-free RPMI 1640, and seeded in the controlmembrane insert on the top portion of the chamber (Corning, USA). The lower compartment of the chamber contained $10 \%$ FBS as a chemo-attractant. After incubated for 24 hour, cells on the membrane were scrubbed, washed with PBS and fixed in methanol and stained with methylrosanilnium chloride solution. For invasive assay, 
the procedures were similar, but we coated the insert with $60 \mu$ l of 1:40 matrigel (Corning, USA).

\section{Statistical analysis}

Results are representative of 3 different experiments, and data are expressed as mean \pm standard deviation. Statistical evaluation was performed using student $t$ test to compare the means of different groups. SPSS 10.0 software was applied to analyze all data and $p<0.05$ was considered statistically significant.

\section{Results}

\section{S100A4 expression in glioma}

Here, we designed S100A4 primers and performed real-time RT-PCR to quantify its mRNA expression level with housekeeping GAPDH as an internal control. The increased S100A4 mRNA expression was observed from glioma in comparison to paired tumor tissue $(p<0.001$, Figure 1A and 1B). Gradually higher S100A4 mRNA expression was observed with glioma grading increased ( $p<0.001$, Figure 1C). Histologically, astrocytoma showed lower S100A4 mRNA expression than oligodendrogliomas, which did lowly in comparison to glioblastomas $(p<0.001$, Figure 1D).

As showed in Figure 2, S100A4 protein was detectable in glioma and matched normal tissue. Densitometric analysis showed S100A4 ovexpression in glioma tissue, compared with matched normal tissue by Western blot $(p<0.001)$. Gradual overexpression of S100A4 was observed when glioma grading became higher

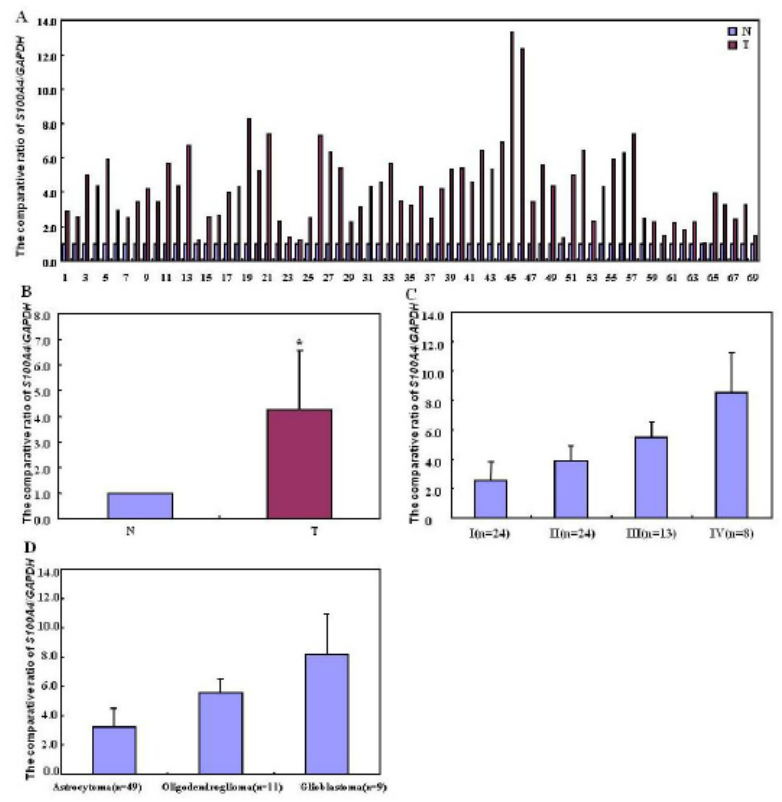

Figure 1.S100A4 mRNA Expression Level in Glioma and its Correlation with Clinicopathological Features of Tumor. S100A4 mRNA was highly expressed in glioma than paired normal brain tissue by real-time RT-PCR (A and B). Its expression level was positively correlated with WHO grading of glioma $(p<0.001, \mathrm{C})$. S100A4 mRNA expression was higher in glioblastoma than oligoglioma $(p<0.001, \mathrm{D})$. Astrocytoma showed S100A4 overexpression in comparison to oligodendrogliomas $(p<0.001, \mathrm{D}) . * p<0.001$. N, normal brain tissue; $\mathrm{C}$, glioma
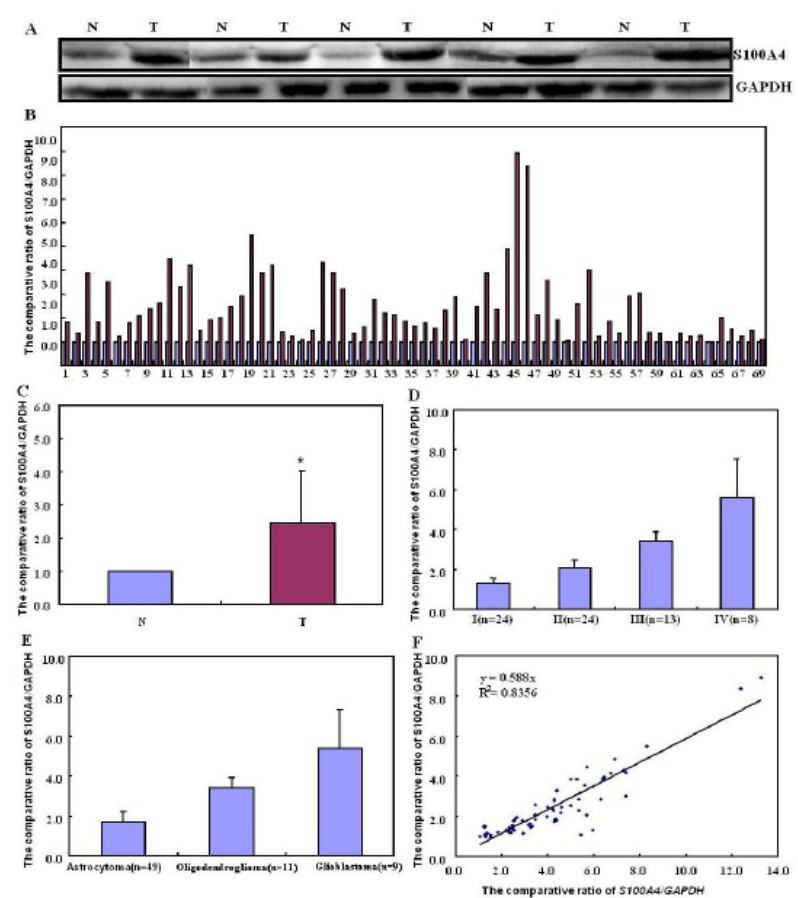

Figure 2. S100A4 Protein Expression Level in Glioma and its Correlation with Clinicopathological Features of Tumor. Cell lysate was loaded and probed with anti-S100A4 $(29 \mathrm{kDa})$ with GADPH $(37 \mathrm{kDa})$ as an internal control A). Densitometric analysis showed that its expression level was increased in glioma, compared with paired normal tissue $(p<0.001, \mathbf{B}$ and C). S100A4 protein expression became gradually stronger with glioma grading higher $(p<0.001, \mathbf{D})$. S100A4 protein was more expressed in glioblastoma than oligodendrogliomas $(p<0.001, \mathrm{E})$. Astrocytoma highly expressed S100A4 in comparison to oligodendrogliomas $(p<0.001, \mathbf{E})$. There was positive relationship between mRNA and protein expression of S100A4 $(p<0.001, \mathbf{F}) .{ }^{*} p<0.001$
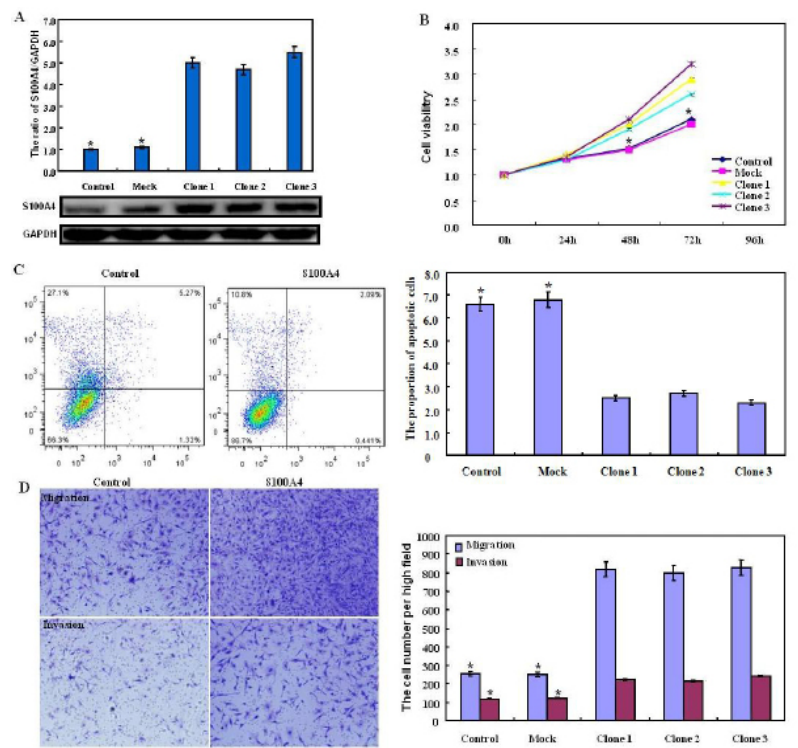

Figure 3. The effects of S100A4 Overexpression on the Phenotypes of Glioma U251 Cells. After transfection of pcDNA3.1-S100A4, S100A4 expression became stronger in three clones than mock and control by real-time RT-PCR and Western blot (A). The transfectants showed a high growth (B), apoptotic suppression (C), a strong ability to migrate and invade (D) in comparison with control and mock. *, $p<0.001$, compared with three transfectant clones 
Ting Jin et al

( $p<0.001)$. According to histological subtyping, lower S100A4 protein expression was detected in astrocytoma than oligodendrogliomas $(p<0.001)$, while the latter did than glioblastoma $(p<0.001)$. There was a positive link between mRNA and protein expression of S100A4 in glioma $(p<0.001)$.

The effects of S100A4 ovexpression on the phenotypes of glioma cells

After transfected with pcDNA3.1-S100A4, U252 cells overexpressed S100A4 at both protein and mRNA levels (Figure 3A). There was a higher growth and lower apoptosis of the S100A4 transfectants than the maternal cells $(p<0.001$, Figure 3B and 3C). Compared with control and mock, forced S100A4 overexpression could enhance migration and invasion of three transfectants by chamber assay $(p<0.001$, Figure 3D).

\section{Discussion}

S100A4 is a member of the S100 family of calciumbinding proteins and possesses a wide range of biological functions, such as regulation of angiogenesis, cell survival, motility, and invasion. Here, we found gradually increased expression of both S100A4 mRNA and protein in glioma, compared with normal brain tissue, which is consistent with other reports about clear cell renal cell carcinoma (Yang et al., 2012), esophageal squamous cell carcinoma (Zhang et al., 2012; Chen et al., 2012), gastric (Zhao et al., 2013) and colorectal carcinoma (Kim et al., 2009), pancreatic (Sekine et al., 2012), breast (Ismail et al., 2008) and bladder cancer (Matsumoto et al., 2007). Liang et al. (2014) found that neutrophil infiltration upregulated S100A4 expression and mediated mesenchymal characteristics of glioma. S100A4 overxpression might be attributable to the hypomethylation of $\mathrm{CpG}$ sites in its first intron or stimulated under the hypoxytic microenvironment (Liu et al., 2010; Horiuchi et al., 2012). Walker et al. (1995) found homozygous deletions of S100A4 gene are rare in non-astrocytic brain tumors. These data indicated that higher S100A4 expression might contribute to the carcinogenesis.

To the role of S100A4 protein in the progression of glioma, its expression was compared with the aggressive behaviors of carcinoma. We found that either S100A4 protein or mRNA expression was positively linked to WHO grading in line with the data from other papers (Feng et al., 2011; Kang et al., 2012). In addition, S100A4 expression is the highest in glioblastoma, lowest in astrocytoma and middle in oligodendrogliomas at both mRNA and protein levels. In other word, aggressive degree of histological subtypes of glioma paralleled with S100A4 expression. According to literature, S100A4 expression was found to correlate with the aggressive parameters of advanced stage endometrial cancer (Tsukamoto et al., 2013) and bladder cancer (Matsumoto et al., 2007). Tsukamoto et al. (2013) reported that S100A4 plays an important role in the invasiveness, particularly with perineural invasion and invasion pattern of pancreatic cancer. It was suggested that S100A4 might be closely linked to aggressiveness of glioma and might be employed to indicate clinicopathological behaviors of glioma in clinical practice.

It was documented that S100A4 could regulate migratory and invasive behavior of human esophageal squamous cell carcinoma cells through modulating AKT/ Slug pathway of EMT (Chen et al., 2012; Zhang et al., $2012 \mathrm{a}$ and $\mathrm{b}$ ). Wnt/ $\beta$-catenin pathway has impact on S100A4- induced cell motility and metastasis of colon cancer cells (Sack et al., 2011). In endometrial cancer cell, S100A4 mediates invasion with the activation of Smads in TGF-beta1 signaling (Xie et al., 2009). S100A4 silencing suppresses proliferation, angiogenesis and invasion of thyroid cancer cells through downregulation of MMP-9 and VEGF (Jia et al., 2013), whereas it induces apoptosis and suppression of cell growth, motility, and invasiveness in pancreatic carcinoma cells (Sekine et al., 2012). In contrast, S100A4 overexpression remarkably accelerates cell motility in pancreatic carcinoma cells (Sekine et al., 2012). In brain tumors, modified expression of S100A4 protein in C6 glioma cells or surrounding astrocytes affects migration of tumor cells (Takenaga et al., 2007) and extracellular S100A4 stimulates the migration rate of astrocytic tumor cells by modifying the organization of their actin cytoskeleton (Belot et al., 2002).

In the present study, ectopic S100A4 overexpression weakened the apoptosis, and enhanced proliferation, migration and invasion of glioma cells in line with previous results (Takenaga et al., 2007; Xie et al., 2009; Sack et al., 2011; Zhang et al., 2012; Jia et al. 2013). Taken together, S100A4 overexpression might worsen the aggressive behaviors and S100A4 silencing could be employed for the gene therapy of malignancies.

In summary, up-regulated expression of S100A4 might play an important role in the histogenesis and subsequent progression of glioma and might be considered as a promising marker to indicate the aggressive behaviors. S100A4 overexpression promotes aggressiveness of glioma so as to be considered as a molecular target of its gene therapy.

\section{References}

Belot N, Pochet R, Heizmann CW, Kiss R, Decaestecker C (2002). Extracellular S100A4 stimulates the migration rate of astrocytic tumor cells by modifying the rganization of their actin cytoskeleton. Biochim Biophys Acta, 1600, 74-83.

Boye K, Maelandsmo GM (2010). S100A4 and metastasis: a small actor playing many roles. Am J Pathol, 176, 528-35.

Chen D, Zheng XF, Yang ZY, et al (2012). S100A4 silencing blocks invasive ability of esophageal squamous cell carcinoma cells. World J Gastroenterol, 18, 915-22.

Dahlmann M, Okhrimenko A, Marcinkowski P, et al (2014). RAGE mediates S100A4-induced cell motility via MAPK/ ERK and hypoxia signaling and is a prognostic biomarker for human colorectal cancer metastasis. Oncotarget, 5, 3220-33.

Feng LZ, Zheng XY, Zhou LX, et al (2011). Correlation between expression of S100A4 and VEGF-C, and lymph node metastasis and prognosis in gastric carcinoma. $J$ Int Med Res, 39, 1333-43.

Horiuchi A, Hayashi T, Kikuchi,N, et al (2012). Hypoxia upregulates ovarian cancer invasiveness via the binding of HIF- $1 \alpha$ to a hypoxia-induced, methylation-free hypoxia response element of S100A4 gene. Int J Cancer, 131, 
1755-67.

Ismail NI, Kaur G, Hashim H, Hassan MS (2008). S100A4 overexpression proves to be independent marker for breast cancer progression. Cancer Cell Int , 8, 12 .

Jenkinson SR, Barraclough R, West CR, Rudland PS (2004). S100A4 regulates cell motility and invasion in an in vitro model for breast cancer metastasis. Br J Cancer, 90, 253-62.

Jia W, Gao XJ, Zhang ZD, Yang ZX, Zhang G (2013). S100A4 silencing suppresses proliferation, angiogenesis and invasion of thyroid cancer cells through downregulation of MMP-9 and VEGF. Eur Rev Med Pharmacol Sci, 17, 1495-508.

Kang YG, Jung CK, Lee A, Kang WK, Oh ST, Kang CS (2012). Prognostic significance of S100A4 mRNA and protein expression in colorectal cancer. J Surg Oncol, 105, 119-24.

Kim JH, Kim CN, Kim SY, et al (2009). Enhanced S100A4 protein expression is clinicopathologically significant to metastatic potential and p53 dysfunction in colorectal cancer. Oncol Rep, 22, 41-7.

Liang J, Piao Y, Holmes L, et al (2014). Neutrophils promote the malignant glioma phenotype through S100A4. Clin Cancer Res, 20, 187-98.

Liu J, Guo Y, Fu S, Yang M, Sun KL, Fu WN (2010). Hypomethylation-induced expression of S100A4 increases the invasiveness of laryngeal squamous cell carcinoma. Oncol Rep, 23, 1101-7.

Louis DN, Ohgaki H, Wiestler OD, Cavenee WK (2007). WHO classification of tumors of the central nervous system, Fourth Edition IARC

Maelandsmo GM, Hovig E, Skrede M, et al (1996). Reversal of the in vivo metastatic phenotype of human tumor cells by an anti-CAPL (mts1) ribozyme. Cancer Res, 56, 5490-8.

Matsumoto K, Irie A, Satoh T, et al (2007). Expression of S100A2 and S100A4 predicts for disease progression and patient survival in bladder cancer. Urology, 70, 602-7.

Mishra SK, Siddique HR, Saleem M (2012). S100A4 calciumbinding protein is key player in tumor progression and metastasis: preclinical and clinical evidence. Cancer Metastasis Rev, 31, 163-72.

Sack U, Walther W, Scudiero D, et al (2011). S100A4-induced cell motility and metastasis is restricted by the $\mathrm{Wnt} / \beta$-catenin pathway inhibitor calcimycin in colon cancer cells. Mol Biol Cell, 22, 3344-54.

Schwartzbaum JA, Fisher JL, Aldape KD, Wrensch M (2006). Epidemiology and molecular pathology of glioma. Nat Clin Pract Neurol, 2, 494-503.

Sekine H, Chen N, Sato K, et al (2012). S100A4, frequently overexpressed in various human cancers, accelerates cell motility in pancreatic cancer cells. Biochem Biophys Res Commun, 429, 214-9.

Simpson PT, Shoker BS, Barraclough R, et al (2003). Examination of tumour histopathology and gene expression in a neu/S100A4 transgenic model of metastatic breast cancer. Int J Exp Pathol, 84, 173-84.

Takenaga K, Nakamura Y, Sakiyama S (1997). Expression of antisense RNA to S100A4 gene encoding an S100-related calcium-binding protein suppresses metastatic potential of high- metastatic Lewis lung carcinoma cells. Oncogene, 14, 331-7.

Takenaga K, Nygren J, Zelenina M, et al (2007). Modified expression of Mts1/S100A4 protein in C6 glioma cells or surrounding astrocytes affects migration of tumor cells in vitro and in vivo. Neurobiol Dis, 25, 455-63.

Trabelsi S, Brahim DH, Ladib M (2014). Glioma epidemiology in the central Tunisian population: 1993-2012. Asian Pac J Cancer Prev, 15, 8753-7.

Tsukamoto N, Egawa S, Akada M, et al (2013). The expression of S100A4 in human pancreatic cancer is associated with ker DG, Duan W, Kaye AH, Lavin MF (1995). Homozygous deletions of the MTS1 gene are rare in non-astrocytic brain tumors. Biochem Biophys Res Commun, 211, 404-9.

Wang H, Duan L, Zou Z, et al (2014). Activation of the PI3K/ $\mathrm{Akt} / \mathrm{mTOR} / \mathrm{p} 70 \mathrm{~S} 6 \mathrm{~K}$ pathway is involved in S100A4-induced viability and migration in colorectal cancer cells. Int $\mathrm{J} \mathrm{Med}$ Sci, 11, 841-9.

Xie R, Schlumbrecht MP, Shipley GL, Xie S, Bassett RL Jr., Broaddus RR (2009). S100A4 mediates endometrial cancer invasion and is a target of TGF-beta1 signaling. Lab Invest, 89, 937-47.

Xu X, Su B, Xie C, et al (2014). Sonic hedgehog-gli1 signaling pathway regulates the epithelial mesenchymal transition (EMT) by mediating a new target gene, S100A4, in pancreatic cancer cells. PLoS One, 9, 96441.

Yang H, Zhao K, Yu Q, Wang X, Song Y,Li R (2012). Evaluation of plasma and tissue S100A4 protein and mRNA levels as potential markers of metastasis and prognosis in clear cell renal cell carcinoma. J Int Med Res, 40, 475-85.

Zhang G,Li M, Jin J, Bai Y, Yang C (2011). Knockdown of S100A4 decreases tumorigenesis and metastasis in osteosarcoma cells by repression of matrix metalloproteinase-9. Asian Pac J Cancer Prev, 12, 2075-80.

Zhang HY, Zheng XZ, Wang XH, Xuan XY, Wang F, Li SS (2012a). S100A4 mediated cell invasion and metastasis of esophageal squamous cell carcinoma via the regulation of MMP-2 and E-cadherin activity. Mol Biol Rep, 39, 199-208.

Zhang K, Zhang M, Zhao H, Yan B, Zhang D, Liang J (2012). S100A4 regulates motility and invasiveness of human esophageal squamous cell carcinoma through modulating the AKT/Slug signal pathway. Dis Esophagus, 25, 731-9.

Zhao Y, Zhang T, Wang Q (2013). S100 calcium-binding protein A4 is a novel independent prognostic factor for the poor prognosis of gastric carcinomas. Oncol Rep, 30, 111-8. 